\title{
17. DEEP SEA DRILLING PROJECT, LEG 1 FORAMINIFERA FROM SELECTED SAMPLES
}

W. H. Blow, British Petroleum Research Centre, London, England

BIOSTRATIGRAPHIC SUMMARY OF AVAILABLE SAMPLES

Hole 1 (lat $25^{\circ} 51.5^{\prime} \mathrm{N}$., long $92^{\circ} 11.0^{\prime} \mathrm{W}$.)

\begin{tabular}{|c|c|c|c|}
\hline \multirow[t]{2}{*}{ Core } & \multicolumn{2}{|c|}{ Depth Below Sea Bed } & \multirow[t]{2}{*}{ Age Determination } \\
\hline & Feet & Meters & \\
\hline 1 & 497 to 527 & 151.5 to 160.5 & $\begin{array}{l}\text { Zone N.22/Zone N.23; } \\
\text { Pleistocene or younger. }\end{array}$ \\
\hline \multicolumn{4}{|c|}{ Hole 2 (lat $23^{\circ} 27.3^{\prime} \mathrm{N}$., long $92^{\circ} 35.2^{\prime} \mathrm{W}$.) } \\
\hline \multirow[t]{2}{*}{ Core } & \multicolumn{2}{|c|}{ Depth Below Sea Bed } & \multirow{2}{*}{ Age Determination } \\
\hline & Feet & Meters & \\
\hline 1 & 64 to 94 & 19.5 to 28.6 & $\begin{array}{l}\text { Upper Zone N.22/Zone N.23; } \\
\text { late Pleistocene or younger. }\end{array}$ \\
\hline 2 & 189 to 219 & 57.6 to 66.7 & $\begin{array}{l}\text { Zone N.22 (?early part); } \\
\text { early Pleistocene. }\end{array}$ \\
\hline 3 & 219 to 233 & 66.7 to 71.0 & $\begin{array}{l}\text { Zone N.20 or Zone N.21 (?latter); } \\
\text { middle to late Pliocene. }\end{array}$ \\
\hline 4 & 333 to 359 & 101.5 to 109.5 & $\begin{array}{l}\text { Zone N.19; } \\
\text { early Pliocene. }\end{array}$ \\
\hline \multicolumn{4}{|c|}{ Hole 3 (lat $23^{\circ} 01.8^{\prime} \mathrm{N}$., long $92^{\circ} 02.6^{\prime} \mathrm{W}$.) } \\
\hline \multirow[t]{2}{*}{ Core } & \multicolumn{2}{|c|}{ Depth Below Sea Bed } & \multirow[t]{2}{*}{ Age Determination } \\
\hline & Feet & Meters & \\
\hline 2 & 117 to 147 & 35.7 to 44.8 & $\begin{array}{l}\text { Zone N.22/Zone N.23; } \\
\text { late Pleistocene or younger. }\end{array}$ \\
\hline 3 & 657 to 687 & 200.3 to 209.7 & $\begin{array}{l}\text { Zone N.22; } \\
\text { probably middle part of Pleistocene. }\end{array}$ \\
\hline 4 & 688 to 718 & 209.7 to 218.8 & $\begin{array}{l}\text { Zone N.22; } \\
\text { part of Pleistocene }\end{array}$ \\
\hline 5 & 1050 to 1080 & 320.0 to 329.1 & $\begin{array}{l}\text { Section 1: ?Zone N.21; } \\
\text { later Pliocene. } \\
\text { Sections 2-5: Zone N.20 or lower } \\
\text { Zone N.21; } \\
\text { middle to late Pliocene. }\end{array}$ \\
\hline 6 & 1083 to 1113 & 330.0 to 339.2 & $\begin{array}{l}\text { Zone N.20 or lower Zone N.21; } \\
\text { middle to late Pliocene. }\end{array}$ \\
\hline 7 & 1250 to 1280 & 381.0 to 390.1 & $\begin{array}{l}\text { Section 2: Zone N.20; } \\
\text { approx. mid-part Pliocene. } \\
\text { Section 3: Zone N.19 or Zone N.20; } \\
\text { approx. mid-part Pliocene. } \\
\text { Section 4: Zone N.19; } \\
\text { early to middle Pliocene. }\end{array}$ \\
\hline
\end{tabular}




\begin{tabular}{cccl}
\hline Core & \multicolumn{2}{c}{ Depth Below Sea Bed } & \multicolumn{1}{c}{ Age Determination } \\
\hline 8 & 1409 to 1439 & 429.5 to 438.6 & $\begin{array}{l}\text { Section 2: early Zone N.19; } \\
\text { early Pliocene. } \\
\text { Sections 3-6: No Determination. }\end{array}$ \\
9 & 1752 to 1782 & 534.0 to 543.2 & $\begin{array}{l}\text { No determination. } \\
\text { (reported as late Miocene by } \\
\text { Berggren ?Zone N.17/N.18.) }\end{array}$ \\
\hline
\end{tabular}

Hole 4 (lat $24^{\circ} 28.7^{\prime} \mathrm{N}$., long $73^{\circ} 47.5^{\prime} \mathrm{W}$.)

\begin{tabular}{cccl}
\hline Core & \multicolumn{2}{c}{ Depth Below Sea Bed } & \multicolumn{1}{c}{ Age Determination } \\
\hline 1 & 0 to 30 & 0 to 9.1 & $\begin{array}{l}\text { Sections 1, 2, 3, 4, 6: No } \\
\text { Determination. }\end{array}$ \\
2 & 342 to 372 & 104.2 to 113.4 & $\begin{array}{l}\text { Section 1: No determination. } \\
3\end{array}$ \\
4 & 639 to 469 & 133.8 to 143.0 & Section 1: No determination. \\
4 & 626 to 656 & 190.8 to 199.9 & Section 1: No determination. \\
\hline
\end{tabular}

\begin{tabular}{|c|c|c|c|}
\hline \multicolumn{4}{|c|}{ Hole $4 \mathrm{~A}$ (lat $24^{\circ} 28.7^{\prime} \mathrm{N}$. , long $73^{\circ} 47.5^{\prime} \mathrm{W}$.) } \\
\hline \multirow[t]{2}{*}{ Core } & \multicolumn{2}{|c|}{ Depth Below Sea Bed } & \multirow[t]{2}{*}{ Age Determination } \\
\hline & Feet & Meters & \\
\hline 1 & 239 to 269 & 72.8 to 81.9 & $\begin{array}{l}\text { Section } 1 \text { : } \\
\text { early to middle Maestrichtian; } \\
\text { Section 2: } \\
\text { early Maestrichtian; } \\
\text { Section 3: } \\
\text { No determination. }\end{array}$ \\
\hline 2 & 375 to 405 & 114 to 123.4 & $\begin{array}{l}\text { Section } 1: \\
\text { No determination. }\end{array}$ \\
\hline
\end{tabular}

Hole 5 (lat $24^{\circ} 43.6^{\prime} \mathrm{N}$., long $73^{\circ} 38.5^{\prime} \mathrm{W}$.)

\begin{tabular}{|c|c|c|c|c|}
\hline \multirow[t]{2}{*}{ Core } & \multicolumn{3}{|c|}{ Depth Below Sea Bed } & \multirow[t]{2}{*}{ Age Determination } \\
\hline & Feet & Met & & \\
\hline 1 & 0 to 30 & 0 to & 9.1 & $\begin{array}{l}\text { Section 1,2,3: } \\
\text { Heterogeneous samples; } \\
\text { Zone N.22/Zone N.23; } \\
\text { late Pleistocene or younger with } \\
\text { derived Neogene and Paleogene. }\end{array}$ \\
\hline 2 & 102 to 132 & 31.1 to & 40.2 & $\begin{array}{l}\text { Section 1: } \\
\text { No determination. }\end{array}$ \\
\hline
\end{tabular}

\begin{tabular}{cccc}
\hline Hole $6\left(\right.$ lat $30^{\circ} 50.4^{\prime} \mathrm{N}$. , long $67^{\circ} 38.9^{\prime} \mathrm{W}$ ) & & \\
\hline Core & \multicolumn{2}{c}{ Depth Below Sea Bed } & Age Determination \\
\hline 2 & 499 to 529 & 152.1 to 161.2 & $\begin{array}{l}\text { Section 1: } \\
\text { No determination. }\end{array}$ \\
\hline
\end{tabular}




\begin{tabular}{|c|c|c|c|}
\hline \multirow[t]{2}{*}{ Core } & \multicolumn{2}{|c|}{ Depth Below Sea Bed } & \multirow[t]{2}{*}{ Age Determination } \\
\hline & Feet & Meters & \\
\hline 3 & 625 to 655 & 190.5 to 199.6 & $\begin{array}{l}\text { Sections } 3,4 \text { : } \\
\text { No determination. }\end{array}$ \\
\hline 4 & 752 to 782 & 229 to 238.4 & $\begin{array}{l}\text { Section 3: } \\
\text { Zone P.11 (=Globigerapsis } \\
\text { kugleri Zone); } \\
\text { earlier part of Lutetian (earlier } \\
\text { Middle Eocene). }\end{array}$ \\
\hline 6 & 817 to 842 & 249.0 to 256.6 & $\begin{array}{l}\text { Section 1: } \\
\text { Zone P.10 (=Hantkenina } \\
\text { aragonensis/Globorotalia } \\
\text { pseudomayeri Zone); } \\
\text { early Middle Eocene (early } \\
\text { Lutetian). }\end{array}$ \\
\hline \multicolumn{4}{|c|}{ Hole 7 (lat $30^{\circ} 08.0^{\prime} \mathrm{N}$., long $68^{\circ} 17.8^{\prime} \mathrm{W}$.) } \\
\hline Core & Feet ${ }^{\text {Dept }}$ & $\begin{array}{l}\text { Sea Bed } \\
\text { Meters }\end{array}$ & Age Determination \\
\hline 11 & 0 to 30 & 0 to 9.1 & $\begin{array}{l}\text { Section 1: Zone N.22/Zone } \\
\text { N.23; } \\
\text { Pleistocene or younger. } \\
\text { Section 2: } \\
\text { No determination. }\end{array}$ \\
\hline
\end{tabular}

\section{FAUNAS OF SAMPLES EXAMINED}

(Determinations by W. H. Blow)

\section{Hole 1}

Sample 1-1-1-2, $75 \mathrm{~cm}$ :

Fairly rich planktonic foraminiferal fauna with Globorotalia (G.) truncatulinoides truncatulinoides; $G$. (G.) truncatulinoides pachytheca; $G$. (G.) tumida tumida; $G$. (G.) cultrata cultrata; $G$. (G.) cultrata menardii; $G$. (G.) ungulata; Globorotalia (Turborotalia) pseudopima; Pulleniatina obliquiloculata finalis; $P$. obliquiloculata obliquiloculata; Globigerinoides conglobatus conglobatus; G. quadrilobatus quadrilobatus; G. quadrilobatus sacculifer (f. typica); G. elongatus; G. miber (red carotene colour present). Globigerina falconensis; G. quadrilatera; G. eggeri eggeri; G. glutinata; G. calida calida; Hastigerina siphonifera siphonifera; $H$. pelagica; Turborotalita spp; Globigerinita spp.

Age: Zone N.23; late Pleistocene/Holocene.

Sample 1-1-1-3, 63-65 cm:

Fairly rich planktonic fauna as above.

Age: Zone N.23; late Pleistocene/Holocene.

Sample 1-1-1-4, 74-76 cm:

Poor planktonic foraminiferal fauna with Orbulina univera; Globigerinoides ruber (red carotene colouration); Globigerinoides quadrilobatus immaturus;
Globigerina eggeri eggeri.

Age: Zone N.22/Zone N.23; Pleistocene or younger.

Sample 1-1-1-5, 74-76 cm:

Poor planktonic foraminiferal fauna with Globorotalia (G.) truncatulinoides truncatulinoides; Hastigerina siphonifera siphonifera; Globigerina falconensis; Globigerinoides elongatus; G. ruber (red carotene colouration), G. quadrilobatus immaturus; Globorotalia (Turborotalia) cf. minutissima, G. (T.) scitula scitula; Globigerinita spp., Turborotalita spp.

Age: Zone N.22/Zone N.23; Pleistocene or younger.

Sample 1-1-1-6, $72-74 \mathrm{~cm}$ :

Fairly rich planktonic fauna with Globorotalia (G.) crassula (s.l.); Globorotalia (G.) truncatulinoides truncatulinoides; $G$. (G.) truncatulinoides pachytheca; $G$. (G.) cultrata cultrata; G. (Turborotalia) crassaformis crassaformis; G. (T.) inflata, Globigerina (Biella) digitata digitata; Globigerina eggeri eggeri; G. glutinata; Globigerinoides conglobatus conglobatus; G. ruber (red carotene colouration); Globigerinoides quadrilobatus sacculifer (f. typica); G. elongatus; Hastigerina siphonifera siphonifera; $H$. pelagica; Orbulina spp., Pulleniatina obliquiloculata obliquiloculata.

Age: Zone N.23; late Pleistocene or younger.

Hole 2

Sample 1-2-1-2, 50-51 cm:

Rich planktonic foraminiferal fauna with Globorotalia 
(G.) truncatulinoides truncatulinoides; $G$. (G.) truncatulinoides pachytheca; $G$. (G.) cultrata menardii; $G$. (Turborotalia) inflata; G. (T.) acostaensis pseudopima; G. (T.) obesa; G. (T.) crassaformis crassaformis; Globigerina rubescens (red carotene colouration); G. falconensis; G. glutinata; Candeina nitida nitida; Pulleniatina obliquiloculata finalis; Orbulina spp.; Globigerinoides conglobatus conglobatus; $G$. ruber (red carotene colouration); G. quadrilobatus sacculifer (f. typica); Hastigerina siphonifera siphonifera; $\mathrm{H}$. pelagica; Globigerinita spp.; Turborotalita spp.

Age: Upper Zone N.22/Zone N.23; late Pleistocene or younger.

Sample 1-2-2-2, 75-76 cm:

As above.

Sample 1-2-2-1, 74-75 cm:

Fairly rich planktonic foraminiferal fauna with Globorotalia (G.) truncatulinoides truncatulinoides (primitive); Sphaeroidinella dehiscens dehiscens; Globorotalia (G.) crassula crassula; G. (G.) cultrata menardii; G. (T.) acostaensis humerosa; G. (T.) acostaensis acostaensis; Globigerina falconensis; G. quadrilobatus; Globigerinoides elongatus; G. cf. bollii; G. quadrilobatus immaturus; Orbulina spp.

Age: Zone N.22 probably early part; early Pleistocene.

Sample 1-2-3-2, $73 \mathrm{~cm}$ :

Rich planktonic foraminiferal fauna with Globorotalia (G.) miocenica; G. (G.) cultrata exilis; G. (G.) crassula conomiozea; $G$. (T.) crassaformis crassaformis; $G$. (T.) acostaensis pseudopima; G. (T.) acostaensis humerosa; $G$. (T.) acostaensis acostaensis; $G$. (T.) minutissima; $G$. (T.) scitula scitula; G. (T.) obesa; Globigerinoides conglobatus conglobatus; G. ruber (white); G. elongatus; G. bollii; G. quadrilobatus immaturus; G. quadrilobațs sacculifer; Obrulina spp.; Hastigerina siphonifera siphonifera; Sphaeroidinella dehiscens dehiscens.

Age: Zone N.20 or Zone N.21 (Probably Zone N.21); middle to late Pliocene.

Sample 1-2-3-3-, 74-75 cm:

Fairly rich planktonic foraminiferal fauna with Globorotalia (G.) miocenica; G. (G.) cultrata exilis; $G$. (G.) crassula conomiozea; $G$. (T.) crassaformis crassaformis; G. (T.) puncticulata; G. (T.) inflata; G. (T.) minutissima; G. (T.) scitula scitula; G. (T.) acostaensis humerosa; $G$. (T.) acostaensis acostaensis; G. (T.) acostaensis pseudopima; Globigerina glutinata; G. falconensis, G. rubescens; Globigerinoides conglobatus conglobatus; G. elongatus; G. ruber (white); G. bollii; G. quadrilobatus immaturus; Orbulina spp.; Hastigerina siphonifera siphonifera; Sphaeroidinella dehiscens dehiscens.

Age: Zone N.20 or Zone N.21; middle to late Pliocene.

Sample 1-2-4-1, $75 \mathrm{~cm}$ :

Rather poor planktonic foraminiferal fauna with
Globoquadrina altispira globosa; G. altispira cf. altispira; Globigerina decoraperta; Globigerinoides elongatus; Globorotalia (G.) tumida tumida; Sphaeroidinella dehiscens dehiscens (f. immaturus); Sphaeroidinellopsis spp.

Age: Zone N19; early Pliocene.

Sample 1-3-4-2, 73-74 cm:

As above.

Age: Zone N.19; early Pliocene.

Hole 3

Sample 1-3-2-3, $75 \mathrm{~cm}$ :

Mainly sand but with sparse planktonic foraminiferal fauna including Globorotalia (G.) truncatulinoides (s.l.); Globigerinoides ruber (red); G. conglobatus conglobatus; Globigerina eggeri eggeri; Globorotalia (T.) acostaensis humerosa.

Age: Zone N.22/Zone N.23; late Pleistocene or younger.

Sample 1-3-3-2, 89.5-90.7 cm:

Rich planktonic foraminiferal fauna with Globorotalia (G.) truncatulinoides (s.l.); G. (G.) tumida tumida; $G$. (G.) cultrata menardii; G. (Turborotalia) inflata; $G$. (T.) scitula scitula; $G$. (T.) trigonula; Globigerina oscitans; G. eggeri eggeri; G. glutinata; G. calida praecalida; Globigerinoides ruber (red and white forms); G. elongatus; G. conglobatus conglobatus; G. quadrilobatus (s.l.); Globigerinita spp.; Turborotalita spp.

Age: Zone N.22; probably middle part of Pleistocene.

Sample 1-3-4-1, $75 \mathrm{~cm}$ :

Rich planktonic foraminiferal fauna with Globorotalia (G.) truncatulinoides truncatulinoides; $G$. (G.) truncatulinoides pachytheca; G. (G.) crassula crassula; $G$. (G.) crassula conomiozea; G. (T.) crassaformis crassaformis; G. (T.) scitula scitula; G. (T.) acostaensis humerosa; G. (T.) acostaensis pseudopima; Globigerina calida praecalida; G. eggeri eggeri; G. quadrilatera; $G$. glutinate; G. falconensis; Globigerinoides conglobatus conglobatus; G. ruber (red); G. elongatus; G. cf. bollii; G. quadrilobatus (s.l.); Orbulina spp.; Hastigerina siphonifera siphonifera; Sphaeroidinella dehiscens dehiscens.

Age: Zone N.22; Pleistocene (part).

Sample 1-3-5-1, $75 \mathrm{~cm}$ :

Rather poor planktonic foraminiferal fauna with Globorotalia (G.) cultrata menardii; $G$. (G.) cultrata exilis; $G$. (T.) crassaformis crassaformis; G. (T.) acostaensis cf. acostaensis; Globigerina decoraperta; G. cf. rubescens (white); Globigerina quadrilatera; Globigerinoides ruber (white); G. elongatus; G. cf. bollii, G. conglobatus conglobatus; Orbulina spp.; Turborotalita spp.

Age: Probably Zone N.21; late Pliocene.

Sample 1-3-5-2, $75 \mathrm{~cm}$ :

Fairly rich planktonic foraminiferal fauna with 
Globorotalia (G.) miocenica; G. (G.) cultrata exilis; $G$. (G.) crassula viola; $G$. ungulata; $G$. (T.) acostaensis pseudopima; G. (T.) acostaensis humerosa; G. (T.) scitula scitula; G. (T.) ventriosa; G. (T.) crassaformis crassaformis; Globigerina bulloides apertura; G. quadrilatera; G. decoraperta; G. pseudofoliata; Globigerinoides conglobatus conglobatus; G. ruber (white); G. elongatus; $G$. cf. gomitulus; $G$. cf. bollii; $G$. obliquus extremus; Orbulina spp.; Turborotalita spp.

Age: Probably Zone N.20 or lower Zone N.21; later Pliocene.

Sample 1-3-5-3, $75 \mathrm{~cm}$ :

As above.

Age: Zone N.20 or lower Zone N.21; later Pliocene.

Sample 1-3-5-4, $75 \mathrm{~cm}$ :

As above.

Age: Zone N.20 or lower Zone N.21; later Pliocene.

Sample 1-3-5-5, 75-76 cm:

As above.

Age: Zone N.20 or lower Zone N.21; later Pliocene.

Sample 1-3-5-1, $75 \mathrm{~cm}$ :

As above.

Age: Zone N.20 or lower Zone N.21; later Pliocene.

Sample 1-3-6-2, 71.5-72.5 cm:

As above.

Age: Zone N.20 or lower Zone N.21; later Pliocene.

Sample 1-3-7-1, $75 \mathrm{~cm}$ :

Fairly rich planktonic foraminiferal fauna with Globorotalia (G.) miocenica; G. (G.) cultrata exilis; G. (G.) crassula viola; $G$. (T.) acostaensis acostaensis, G. (T.) acostaensis pseudopima (rare); G. (T.) acostaensis humerosa; Sphaeroidinella dehiscens dehiscens; Sphaeroidinellopsis seminulina seminulina; Globigerina bulbosa; G. quadrilatera; G. cf. eggeri eggeri; G. praebulloides occulsa; Globoquadrina larmeni obesa; G. altispira cf. globosa; Globigerinoides conglobatus conglobatus; G. ruber (white); G. quadrilobatus (s.l.); G. elongatus; Orbulina spp.; Hastigerina siphonifera siphonifera; Turborotalita spp.

Age: Zone N.20; middle Pliocene.

Sample 1-3-7-2, $85 \mathrm{~cm}$ :

As above.

Age: Zone N.20; middle Pliocene.

Sample 1-3-7-3, $75 \mathrm{~cm}$ :

Rather poor planktonic foraminiferal fauna with Sphaeroidinella dehiscens dehiscens (including forma immatura); Globorotalia (G.) miocenica; $G$. (G.) tumida tumida; G. (G.) cultrata exilis; G. (T.) acostaensis acostaensis; G. (T.) crassaformis crassaformis; Globigerina eggeri eggeri; Globigerinoides quadrilobatus (s.l.); $G$. ruber (white); G. elongatus. Orbulina sp.; Turborotalita spp.

Age: Zone N.19 or Zone N.20; Pliocene (approx. middle part).

Sample 1-3-7-4, 73-74 cm:

Fairly rich planktonic foraminiferal fauna with Globorotalia (G.) tumida tumida; G. (G.) miocenica; G. (G.) cultrata menardii; $G$. (G.) crassula viola; $G$. (G.) crassula conomiozea; G. (T.) crassaformis crassaformis; G. (T.) crassaformis ronda; G. (T.) scitula scitula; G. (T.) acostaensis acostaensis; G. (T.) acostaensis humerosa; Sphaeroidinella dehiscens dehiscens f. immatura;Sphaeroidienllopsis aubdehiscens paenedehiscens, S. seminulina; Globigernia nepenthes (v. rare); G. eggeri cf. eggeri; Globigerinoides conglobatus conglobatus; G. ruber; $G$. quadrilobatus (s.l.); G. elongatus; Orbulina spp.; Turborotalita spp.

Age: Zone N.19; early to middle Pliocene.

Sample 1-3-8-1, 90-91 cm:

No planktonic foraminifera.

Sample 1-3-8-2, 75-76 cm:

Rather poor planktonic foraminiferal fauna with Spaeroidinella dehiscens dehiscens forma immatura (v. rare); Sphaeroidinellopsis subdehiscens paenedehiscens; Globorotalia (G.) miocenica; G. (G.) margaritae; G. (G.) cf. hirsuta praehirsuta; Globigerina nepenthes; $G$. cf. eggeri multiloba; Orbulina sp., Turborotalita sp.

Age: early Zone N.19; early Pliocene.

Sample 1-3-8-3, $75 \mathrm{~cm}$ :

No planktonic foraminifera

Sample 1-3-8-4, 71-72 cm:

No planktonic foraminifera.

Sample 1-3-8-5, 75-76 cm:

No significant planktonic foraminifera.

Sample 1-3-8-6, $75 \mathrm{~cm}$ :

No significant planktonic foraminifera.

Sample 1-3-9-4, $77 \mathrm{~cm}$ :

No planktonic foraminifera.

Sample 1-3-9-7, 74-75 cm:

No planktonic foraminifera.

\section{Hole 4}

Sample 1-4-1-1, $75 \mathrm{~cm}$ :

No planktonic foraminifera.

Sample 1-4-1-2, $75 \mathrm{~cm}$ :

No planktonic foraminifera. 
Sample 1-4-1-3, $75 \mathrm{~cm}$ :

No planktonic foraminifera.

Sample 1-4-1-4, $75 \mathrm{~cm}$ :

No planktonic foraminifera.

Sample 1-4-1-6, $75 \mathrm{~cm}$ :

No planktonic foraminifera.

Sample 1-4-2-1, 94-96 cm:

No planktonic foraminifera.

Sample 1-4-3-1, 69-71 cm:

No planktonic foraminifera.

Sample 1-4-4-1, 77-80 cm:

No planktonic foraminifera.

\section{Hole 4A}

Sample 1-4A-1-1, 75-76 cm:

Rich planktonic foraminiferal fauna with Globotruncana arca; $G$. cf. calciformis; G. fornicata; G. elevata stuartiformis, G. cf. aegyptica; G. linneiana; G. ackermanni (group); Pseudotextularia spp.

Age: Early to middle Maestrichtian.

Sample 1-4A-1-2, $75 \mathrm{~cm}$ :

Globotruncana cf. arca; G. fornicata; G. elevata stuartiformis; G. stuarti; G. linneiana. (Note also larger foraminifera Vaughanina cubensis, det. W. J. Clarke). Age: Early Maestrichtian.

Sample 1-4A-1-3, 74-75 cm:

No significant planktonic foraminifera.

Sample 1-4A-2-1, 74-76 cm:

No recognizable planktonic foraminifera.

\section{Hole 5}

Sample 1-5-1-1, 74-76 cm:

Rather poor planktonic foraminiferal fauna with Globorotalia (G.) truncatulinoides pachytheca;Globigerinoides conglobatus conglobatus; G. ruber (red); Globigerina rubescens; Truncorotaloides sp.

Age: Zone N.22/Zone N.23; derived Eocene (Pleistocene or younger, with reworked Paleogene).

Sample 1-5-1-2, $100 \mathrm{~cm}$ :

Rather poor planktonic foraminiferal fauna, specimens rolled and abraded, with Globigerina angiporoides, Globigerinita martini scandretti; Truncorotaloides rohri (group); Globigerinoides ruber; Globigerinoides quadrilobatus (s.l.).

Age: Heterogeneous sample, Eocene \& early Oligicene reworked into younger(?latePliocene/Pleistocene)

Sample 1-5-1-3, $74 \mathrm{~cm}$ :

Rather poor planktonic foraminiferal fauna with larger foraminifera including Miogypsina; Lepidocyclina, $\mathrm{Pa}$ laeonummulites; ?Dictyoconus. P.F. includes specimens referable to Globigerinoides ruber; $G$. quadrilobatus; $G$. elongatus; Truncorotaloides sp.; Globigerina ampliapertura; G. prasaepis; Globoquadrina altispira cf. globosa.

Age: Heterogeneous sample. ?late Pliocene/Pleistocene with derived Miocene, Oligocene and Eocene.

Sample 1-5-2-1, 58-60 cm:

No determinable fauna.

\section{Hole 6}

Sample 1-6-2-1, 74-76 cm:

No foraminifera.

Sample 1-6-3-3, 77-79 cm:

No foraminifera.

Sample 1-6-3-4, 88-91 cm:

Rich assemblage of sponge spicules and Radiolaria. No foraminifera.

Age: None

Sample 1-6-4-3, 66-68 cm:

Rich planktonic foraminifera fauna with Globigerina boweri; G. frontosa; Acarinina densa; Globigerina (?) linaperta; Truncorotaloides rohri (group); Globigerina (?) senni; Globigerapsis index; Globotruncana spp.

Age: Zone P.11, /Globigerapsis kygleri Zone, of Bolli, 1957) with Upper Cretaceous (=earlier part of Lutetian, Middle Eocene with derived Upper Cretaceous).

Sample 1-6-6-1, 72-75 cm:

Rich planktonic foraminiferal fauna with Globorotalia broedermanni; Acarinina densa; Globigerina (?) linaperta; Globigerina boweri; G. cf. frontosa; Globigerina (?) senni; Globorotalia pseudomayeri; Globigerinoides (?) higginsi.

Age: Zone P.10 (=Hantkenina aragonensis/Globorotalia pseudomayeri Zone); early Middle Eocene (i.e. early Lutetian).

\section{Hole 7}

Sample 1-7-1-1, 74-76 cm:

Rich planktonic fauna with Globorotalia (G.) truncatulinoides (s.l.); G. (G.) hirsuta hirsuta; G. (G.) cultrata menardii; Hastigerina pelagica; Globigerinoides conglobatus conglobatus; Globigerinoides ruber; Globorotalia (T.) inflata; $G$. (T.) acostaensis pseudopima; $G$. (T.) acostaensis humerosa; Globigerina eggeri eggeri; G. falconensis; Orbulina spp.

Age: Zone N.22/Zone N.23; Pleistocene or younger.

Sample 1-7-1-2, $91-93 \mathrm{~cm}$ :

No significant planktonic foraminiferal species. 